\title{
Research on the Social Security Fund Risk Control and Management Measures Based on Cloud Audit and Big Data Era
}

\author{
Huizhen Long ${ }^{1}$, Tong $\mathrm{Yi}{ }^{\mathrm{2}}$ \\ ${ }^{1}$ Columbia University, New York 10026, USA \\ ${ }^{2}$ Guangxi Normal University, Guilin 541001, China \\ Email: h12998@columbia.edu
}

\begin{abstract}
Affected by the continuous development of China's national economy and other aspects, the number of people participating in social security and the amount of money in China have continued to expand. As a result, the scale of social security fund financial data will continue to expand. In such cases, traditional auditing techniques are often used to standardize the data. With the continuous development of big data technology and cloud audit technology, they have provided corresponding technical support for the development of China's social security fund audit technology. However, the risks involved cannot be ignored. This paper is based on the social security fund system in the era of cloud auditing and big data. Based on the analysis of the problems, it puts forward corresponding measures on how to better control and manage the risks in order to provide corresponding management reference for the financial data risk management control of social security funds in China.
\end{abstract}

Keywords: cloud audit, big data, social security fund, risk control, problems, management measures

\section{Analysis of social security fund system based on cloud audit and big data technology}

\subsection{Data collection summary}

The data collection, payment, management, etc. of social security funds in various regions and related documents are collected and summarized by the audited ports. For example, the total income of the five social insurance funds in 2016 was $5,536.3$ billion yuan, an increase of 755.1 billion yuan or $16.4 \%$ over the previous year. The total fund expenditure was 4,688.8 billion yuan, an increase of 790 billion yuan over the previous year, and an increase of $20.3 \%$. The social security participation numbers and completeness of insurance information in these data are the most critical issues that need to be focused on. In the process of collecting these raw data, the audited unit needs to make corresponding backup work for these data, and strictly monitor the operation of the operator to prevent the situation of artificial deletion of data. After that, using the cloud interface technology enables you to upload these raw data to the cloud, so that the audit department can carry out the subsequent inspection and supervision.

\subsection{Data processing}

After the audited unit uploads the original data to the cloud, the cloud needs to perform the processing of the original data in accordance with the previously set procedures. Since the processing of these data are all transferred to the cloud, it is also called cloud audit. The specific workflow is as follows.

First, clean up and process the original data. Starting from different social security fund characteristics and data storage conversion formats, these data are uniformly converted into standardized data that meets the needs of the audit terminal, and then proceed from the pre-seting audit plan in order to finally form a basis to meet audit needs and analysis form. Second, analyze the original primitive data work. With the help of various audit models pre-seting in the cloud, the original data, foundation, and analysis forms of the social security fund are automatically and intelligently analyzed. But when the valid, invalid, and suspicious data are found, the corresponding backup work is conducted under the premise of collecting the analysis report. Third, deal with the storage of data. The data here mainly include raw data after processing and analyzing. At the same time, the application of cloud auditing also solves the problem of the ports being audited using hardware to store massive data.

\subsection{Call of data}

The staff of the audit terminal can call the audit data and audit model in the cloud after logging in to the cloud audit platform through various types of mobile terminals. With the data analysis and calculation function of cloud audit, the data 
calculation and processing burden of the audit terminal is greatly reduced, which greatly improves the work efficiency of the audit operation.

\section{Analysis of risk control of social security fund based on cloud audit and big data}

\subsection{Risks in initial data statistics}

The preliminary data is an important source of the original data of all social security funds. In other words, the overall quality and quantity of the initial data have a very important impact on the quality of cloud audit work. For example, by 2050, the number of Chinese elderly aged 60 and over will reach 450 million and 336 million, accounting for 32.7\% and $24.4 \%$ of the total population, respectively. In the statistics of the social security fund, whether the collection of these initial data is complete or not is particularly important. However, due to the large number of businesses in the statistical work of China's social security funds and the relatively wide scope involved, coupled with the large amount of content involved and the relatively limited number of staff members, it is very easy to miss the number of participants. 1. The completeness of the insured information of the insured personnel is low, the insured enterprise fails to pay or partially pays the insured fund, and the insured enterprise has an unreasonable payment base and payment rate. These problems are common risks in the initial data collection and aggregation process. The existence of these risks causes a large error in the accuracy and completeness of the initial data, which will directly lead to a decline in the quality of subsequent terminal audit data. .

\subsection{The risks of data upload}

Based on the cloud audit and big data technology, the social security fund data audit work is carried out by means of external networks in the process of data aggregation. Taking Shandong Province as an example, in November 2017, the number of employees participating in the pension, employee medical care, unemployment, work injury, and maternity insurance programs in the province reached 26.526 million, 20.068 million, 12.642 million, 15.318 million, 11.789 million people; 45.293 million residents and 72.725 million people are covered by the pension and resident medical insurance programs, ranking second in the country. Facing the huge social security data, the external network is still the main channel in the process of data processing and uploading. However, in the process of uploading data from external networks, problems such as incomplete data transmission and data upload failures will occur. Even some companies will be attacked by viruses and hackers in the process of uploading the original data, leading to data leakage problems. The existence of these problems has brought great risks to the subsequent social security fund data audit work. At the same time, in the process of uploading data, the security and stability in the process of logging in and accessing the audit platform is completely based on the security built into the basic API. As long as there are insecure factors within the API, they will bring the corresponding data uploading risks.

\subsection{The integrity of the application system is poor}

As far as the current situation is concerned, China's social security fund audit work system still has its own phenomenon, although in some areas, all the insured personnel's insured information is stored and used in a centralized manner. However, there are corresponding inconsistencies in the system and data standards of various departments, and there are also large differences in the specific ideas, methods, and means of management, which leads to the way of data communication and sharing. There are major limitations. For example, even if the social security department and the public security and judicial departments regularly carry out data comparison work, there will be some lags in the extraction and comparison of data, which will lead to various social security funds for some prisoners and deaths. They will cause the relevant departments to spend a lot of time and costs to recover this part of the funds.

\subsection{Cloud audit itself is vulnerable}

Based on cloud audits and big data, the social security fund audits are performed on the basis of external networks during the initial data collection, collation, entry, and summary. However, because network technologies have not yet fully developed and matured under certain circumstances, all external networks are more or less at risk. The openness and sharing of the network resources themselves make the data of the Social Security Center very vulnerable to illegal intrusions and attacks such as viruses and hackers. This is also one of the sources of risk for the audit work of the Social Security Cloud Fund. In addition, these aggregated and collected raw data are saved and organized with the help of electronic data. As long as they are attacked from the external network, it is very easy to cause the electronic database of the Social Security Center to be paralyzed or even collapsed. It has greater difficulty, which greatly increases the risk of audit work. 


\section{Management measures based on cloud audit and big data social security fund risk control}

\subsection{Architecture and improvement of coud security defense system}

For improving the security of social security cloud audit work, the cloud security defense system is the key to it. The security and stability of its operation is of great significance for reducing the risk of cloud audit work and improving the work efficiency. A highly complete security monitoring system is mainly composed of firewall technology, illegal intrusion and rhino-virus protection technology. Specifically, we need to focus on the implementation of the following measures: First, the application of physical isolation technology. In order to better ensure the security of the professional network of cloud auditing, corresponding internal professional security protocols and encryption verification can be adopted to ensure strict identity verification for various types of login requests. Second, the structure and improvement of the security monitoring system. The Social Security Center can start from the shortcomings of the current network security system, cooperate with some strong network security companies, and start with a comprehensive security structure based on the actual security requirements of the monitoring system Third, regular inspections of the safety monitoring system. The current security system has a certain effect on the interception of some external network attacks. However, with the continuous development of related technologies, it has not been able to effectively block emerging virus and hacker attacks. Auditing brings new risks. To this end, it is necessary to make corresponding system resource cleanup and effective release of the operating system for the advanced security monitoring system, to minimize the risk of system crash, and to apply the technical vulnerability detection technology to improve the security and stability of the server promotion.

\subsection{Further integration of application systems}

The future development of big data technology will further bring about the integration and improvement of the social security system, improve the data synergy capability, and support of relevant policies, so that the data update speed of the cloud audit platform can be effectively improved. At the same time, it is also necessary to eliminate the barriers existing between various departments and further improve the interoperability between data, so that the correlation between data is greatly improved, and the previous fund recovery work will be transformed to prevent and control risks. By improving the integration of the application system, the security of the social security fund and its data can be better protected at the source.

\subsection{Further improvement of data transmission security}

In order to ensure the improvement of data security, it is necessary to use data private network transmission and encryption control technology to minimize the danger of data transmission. Through the application of data private network transmission technology, the probability of being attacked from the external network during data transmission is minimized. Using data transmission engineering in the operation of the system's internal network, the external network virus can be implanted and hacked. The risk of the attack is minimized, and the first insurance is added to the transmission of data. In addition, the encryption control of data is the second insurance measure in the overall data transmission. With the application of encryption technology, the corresponding password conversion and transmission can be carried out on the original data, and only after the conversion by a specific password. All raw data can be obtained. Through the use of these two methods, the overall data transmission security is greatly improved.

\subsection{Enhancement of access control technology}

In the process of auditing and the audited terminal's use of cloud audit to carry out corresponding serial operations, it is necessary to use unified identity authentication to well control the user's login and access permissions. In the process of authenticating login personnel, you can use the dual password management method. In the process of logging in to the cloud audit platform, in addition to setting a fixed login password, you also need to set a corresponding dynamic reminder and unique identification password (such as face or fingerprint). Through the application of these methods, illegal login behaviors such as illegal users or fraudulent use of usernames can be resisted, and the risk of illegal downloading and modifying data in the cloud can be minimized.

\section{Conclusion}

With the continuous development of China's social security industry, traditional auditing technology cannot meet the auditing needs of large-scale fund data. Due to the continuous development of cloud platforms and big data technology, cloud auditing technology has been gradually applied to the auditing of social security funds. There are still many risks 
in the initial data transmission and other aspects. To this end, it is necessary to improve the security of the overall social security fund audit system through measures such as the improvement of the cloud security defense system and the further integration of application systems.

\section{References}

[1] Liu Jianhong. Research on the effective docking of social security fund safety assessment and medical insurance supervision. Journal of Aerospace Medicine. 2019; 30(09): 1037-1038.

[2] Ren Weihai. Discussion on Risk Management and Internal Control of Social Security Funds. Accounting of China Township Enterprises. 2019; 09: 247-248.

[3] Du Lili. Research on Risk Prevention and Control System of Social Security Fund. Taxation. 2019; $13(24)$ : 189.

[4] Yang Caihong. Thinking about Strengthening the Risk Prevention and Control of Social Insurance Funds. Sichuan Labor Security. 2019; 08: 23.

[5] Ling Xiang. Thinking about the Risk Management and Internal Control of Social Security Funds. Modern Economic Information. 2019; 15: 133.

[6] Zhu Dejin. Research on Risk Prevention and Control System of Social Security Fund. Learning of Finance and Accounting. 2018; 26: 192.

[7] Zhang Ying. Discussion on Risk Management and Internal Control of Social Security Funds. Finance and Economics (Academic Edition). 2018; 16: 139.

[8] Zhang Mingrui. Risk Management and Internal Control of Social Security Funds. Finance and Economics (Academic Edition). 2017; 20: 138.

[9] Pan Bin. Thinking about Strengthening the Accounting of Social Security Foundation. China Collective Economy. 2017; 17: 96-97.

[10] Zheng Zhepeng. Research on Risk Prevention and Control of Guiyang Medical Insurance Fund Based on Data Mining Technology. Guizhou University of Finance and Economics; 2017. 